\title{
Predictive and prognostic value of phosphorylated c-KIT and PDGFRA in advanced non-small cell lung cancer harboring ALK fusion
}

\author{
HAIHONG YANG ${ }^{1}$, FENGNAN WANG $^{1}$, QIUHUA DENG $^{2}$, DAKAI XIAO ${ }^{2}$, \\ PING HE ${ }^{3}$, XIAODONG LIN ${ }^{3}$ and DONGYUN HE ${ }^{1}$ \\ ${ }^{1}$ Department of Thoracic Oncology; ${ }^{2}$ Centre for Translational Medicine; ${ }^{3}$ Department of Pathology, \\ The First Affiliated Hospital, Guangzhou Medical University, Guangzhou, Guangdong 510120, P.R. China
}

Received May 3, 2018; Accepted November 11, 2018

DOI: $10.3892 / \mathrm{ol} .2019 .9972$

\begin{abstract}
Secondary KIT gene amplification leads to tyrosine kinase inhibitor resistance in anaplastic lymphoma kinase (ALK) fusion-positive advanced non-small cell lung cancer (NSCLC). The presence of the 4q12 amplicon causes the activation of downstream mast/stem cell growth factor receptor Kit (c-Kit) or platelet-derived growth factor receptor $\alpha$ (PDGFRA) signaling pathways. Therefore, in the present study, the association between the functional proteins phosphorylated c-Kit (p-c-Kit) and phosphorylated PDGFRA (p-PDGFRA) and the prognosis of ALK fusion NSCLC was investigated. Advanced stage NSCLC samples with ALK fusion were tested for their p-c-Kit and p-PDGFRA content by immunohistochemical staining, and for its association with crizotinib efficacy and the survival of the patients. Of 64 eligible ALK-positive patients with NSCLC, 30 (46.9\%) were p-c-Kit-positive and 10 (15.7\%) were p-PDGFRA-positive. Brain metastases were more common in ALK-positive cases that were p-PDGFRA-positive compared with those who were p-PDGFRA-negative. ALK-positive patients treated with crizotinib, who exhibited high levels of p-c-Kit had significantly lower progression-free survival times than those with low levels. In addition, the patients with high levels of p-c-Kit exhibited lower overall survival times than those with low levels. Furthermore, multivariate analysis indicated that high levels of p-c-Kit in patients with ALK fusion was the only significant predictive factor for crizotinib efficacy and was a prognostic factor for poor overall survival time. However, no
\end{abstract}

Correspondence to: Dr Haihong Yang, Department of Thoracic Oncology, The First Affiliated Hospital, Guangzhou Medical University, 151 Yanjiang Road, Guangzhou, Guangdong 510120, P.R. China

E-mail: bjrf2009@yahoo.com

Key words: anaplastic lymphoma kinase, mast/stem cell growth factor receptor Kit, platelet-derived growth factor receptor $\alpha$, non-small cell lung cancer, intrinsic resistance statistically significant difference was observed in the survival of patients with different p-PDGFRA levels. p-PDGFRA was more frequently expressed in the ALK-positive cases with brain metastasis. c-Kit signaling activation may be associated with poor efficacy of crizotinib and poor prognosis in advanced ALK fusion NSCLC.

\section{Introduction}

Anaplastic lymphoma kinase (ALK)-fusion genes, including encoding echinoderm microtubule-associated protein-like 4 (EML4)-ALK fusion variant, represent a small but important fraction of oncogenic driver mutations in non-small cell lung cancer (NSCLC), accounting for approximately 3-7\% in all cases worldwide $(1,2)$. Small molecule tyrosine kinase inhibitors (TKIs) are part of the standard therapy for ALK fusion NSCLC. Resistance to first-generation TKI crizotinib within the first 2 years following treatment is mediated by a variety of mechanisms, including secondary mutations within the ALK tyrosine kinase domain and activation of alternative signaling pathways, including those involving the $A L K$ fusion gene or secondary KIT gene amplification (3).

Single nucleotide polymorphism (SNP) array data on NSCLC tissues and cell lines were evaluated for copy number aberrations, and amplification of chromosomal segment $4 \mathrm{q} 12$ overlapping the locus of proto-oncogenes PDGFRA and KIT was observed in $4.2 \%$ NSCLC samples (4). Therefore, in the present study it was also taken into consideration whether there may be an activation of the c-Kit/PDGFRA pathway in the ALK-fusion tumor at the initial stage of NSCLC, which may subsequently lead to intrinsic TKI resistance.

The phosphorylated forms of c-Kit and PDGFRA were selected as biomarkers because phosphorylated proteins are the biologically active states that function within the cell. In order to gain comprehensive understanding of the phosphorylated functional proteins in the c-Kit/PDGFRA signaling pathway and their association with clinicopathological characteristics of patients with ALK fusion, the expression of p-c-Kit and p-PDGFRA were investigated, along with their association with the clinical outcomes of patients with advanced stage NSCLC with ALK fusion. 


\section{Patients and methods}

Patients and samples. Patients with tumors that were ALK-positive, as detected by immunohistochemical staining (IHC) at The First Affiliated Hospital of Guangzhou Medical University between January 2012 and March 2017, were selected retrospectively for the present study. The tumors were staged pathologically according to the 2009 International Association for the Study of Lung Cancer (version 7) (5). Clinical responses were evaluated 1 month following the first administration of ALK-TKI (crizotinib) (250 mg twice daily) and then every 3 months using computed tomography or magnetic resonance imaging scans. The final follow-up time point was in May 2017. The objective response rate (ORR) and disease control rate (DCR) were assessed independently by the present investigators and one radiologist, according to the Response Evaluation Criteria In Solid Tumors (RECIST version 1.1) (6). Progression-free survival (PFS) was measured from the day of treatment initiation until disease progression or mortality. Overall survival (OS) time was measured from the day of initiated treatment until death. Formalin-fixed and paraffin-embedded (FFPE) primary tumor tissues collected during bronchoscopic or percutaneous lung biopsies were evaluated by two pathologists in order to meet the criterion of $\geq 50 \%$ tumor cells. Specimens of insufficient tissue quantity or quality for molecular analyses were excluded. The present study was approved by the Institutional Review Board of The First Affiliated Hospital, Guangzhou Medical University. Written informed consent was obtained from all participants prior to the study.

IHC staining. FFPE NSCLC tissue specimens from patients with metastatic NSCLC were prospectively tested for ALK by IHC using the Ventana platform (Roche Diagnostics, Basel, Switzerland). The assay was developed as a system with the Ventana anti-ALK (D5F3) rabbit monoclonal primary antibody (dilution, 1:100; cat. no., Ref 790-4794; Roche Diagnostics, Basel, Switzerland), according to the manufacturer's protocols, in combination with the OptiView DAB IHC detection and OptiView Amplification kits (Ventana Medical Systems, Inc, Tucson, AZ, USA) for use on a Ventana BenchMark XT automated staining instrument (Ventana Medical Systems, Inc.).

ALK-positive tumor FFPE sections (4 mm thick) were used for IHC using an automated immunostainer (Leica Microsystems, Germany). Briefly, the slides were heated at $95^{\circ} \mathrm{C}$ for $10 \mathrm{~min}$ for antigen retrieval, and endogenous biotin was blocked at room temperature for 10 min using a endogenous biotin blocking kit (cat. no., ab64212, Abcam, Cambridge, UK), and the assay was performed according to the manufacturer's protocols. Following incubation at $4^{\circ} \mathrm{C}$ overnight with anti-c-Kit (phosphor Y703) (dilution, 1:50; ab62154) or anti-PDGFRA (phosphor Y754) (dilution, 1:100; ab5460) antibody obtained from Abcam. The sections were subsequently incubated with biotinylated secondary anti-rabbit antibodies with 1:500 dilution (cat. no., K500711) with LSAB2 system-HRP (DAKO; Agilent Technologies, Inc, Santa Clara, CA, USA) for $30 \mathrm{~min}$ at room temperature. The stained specimens were analyzed by two independent pathologists using an fluorescent Olympus BX51 microscope (magnification x200-1,000; Olympus Corporation,
Tokyo, Japan) and results were scored according to a semi-quantitative method (7) to reveal the staining intensity $(0=$ negative; $1=$ weak $/$ trace; $2=$ moderate; and $3=$ strong $)$ and the percentage of positive cells $(0, \leq 10 ; 1,11-25 ; 2,26-50 ; 3$, $51-75$; and $4,76-100 \%$ ). This grading produced a final score of $0-7$, calculated as the sum of intensity and percentage scores. Therefore, the IHC staining was reported as negative (score $0-1$ ) or positive (score 2-7), further classified as weak (score 2-3), moderate (score 4-5) or strong (score 6-7). In c-KIT staining low protein expression was defined as having negative or weak IHC staining, and high expression was defined as having moderate or strong staining.

Statistical analysis. The $\chi^{2}$ or Fisher exact tests were used to compare categorical variables. The Kaplan-Meier method was used to calculate the PFS and OS rates, and the log-rank test was performed to compare the PFS and OS among the groups. Cox multivariate proportional hazard model was used for survival analysis, and the hazard ratio (HR) and 95\% confidence interval (CI) were calculated using SPSS 16.0 (IBM Corp., Armonk, NY, USA). $\mathrm{P}<0.05$ was considered to indicate statistically significant differences in a 2-way analysis.

\section{Results}

Patient characteristics. Samples from 64 patients with advanced ALK fusion NSCLC were selected. The patients had, a median age of 52 years (range, 25-81 years). Histologically, 62 samples classified as adenocarcinoma and 2 as adenosquamous carcinoma. Brain metastasis had occurred in 26 (40.6\%) patients, including $20(76.9 \%)$ patients at initial diagnosis and $6(23.1 \%)$ patients while receiving crizotinib treatment. Within this cohort, 41 patients had received crizotinib treatment. The characteristics of the patients are outlined in Table I.

Expression of p-c-Kit and p-PDGFRA in ALK fusion NSCLC tumor samples. The levels of p-c-Kit and p-PDGFRA were detected in the samples of tumors with ALK fusion. IHC on the tumor cells revealed that p-c-Kit was mainly expressed in the membrane and cytoplasm (Fig. 1A), and p-PDGFRA was mainly detected in the membrane (Fig. 1B). The associations between p-c-Kit and p-PDGFRA levels and the clinicopathological features of the patients with advanced NSCLC are depicted in Table I. Out of 64 ALK fusion tumor samples, 30 (46.9\%) were p-c-Kit-positive. High levels of p-c-Kit were observed in 16 out of 64 (25\%) tumors. The tumors from older patients ( $\geq 52$ years) exhibited high p-c-Kit expression significantly more frequently than those from younger individuals $(\mathrm{P}=0.03)$. Regarding p-PDGFRA expression, 11 out of 64 (17.2\%) specimens were positive. Positivity for p-PDGFRA was observed with a higher occurrence in the samples of ALK fusion patients with brain metastasis than those without (34.6 vs. $5.3 \%$; $\mathrm{P}=0.01)$.

Association between p-c-Kit and p-PDGFRA expression and the efficacy of crizotinib. In the total study population, the ORR (the sum of complete and partial response rate) and DCR (the sum of complete, partial response and stable disease rate) were 68.2 and $90.2 \%$, respectively (Table I). There was no difference in either ORR or DCR between high-level and 


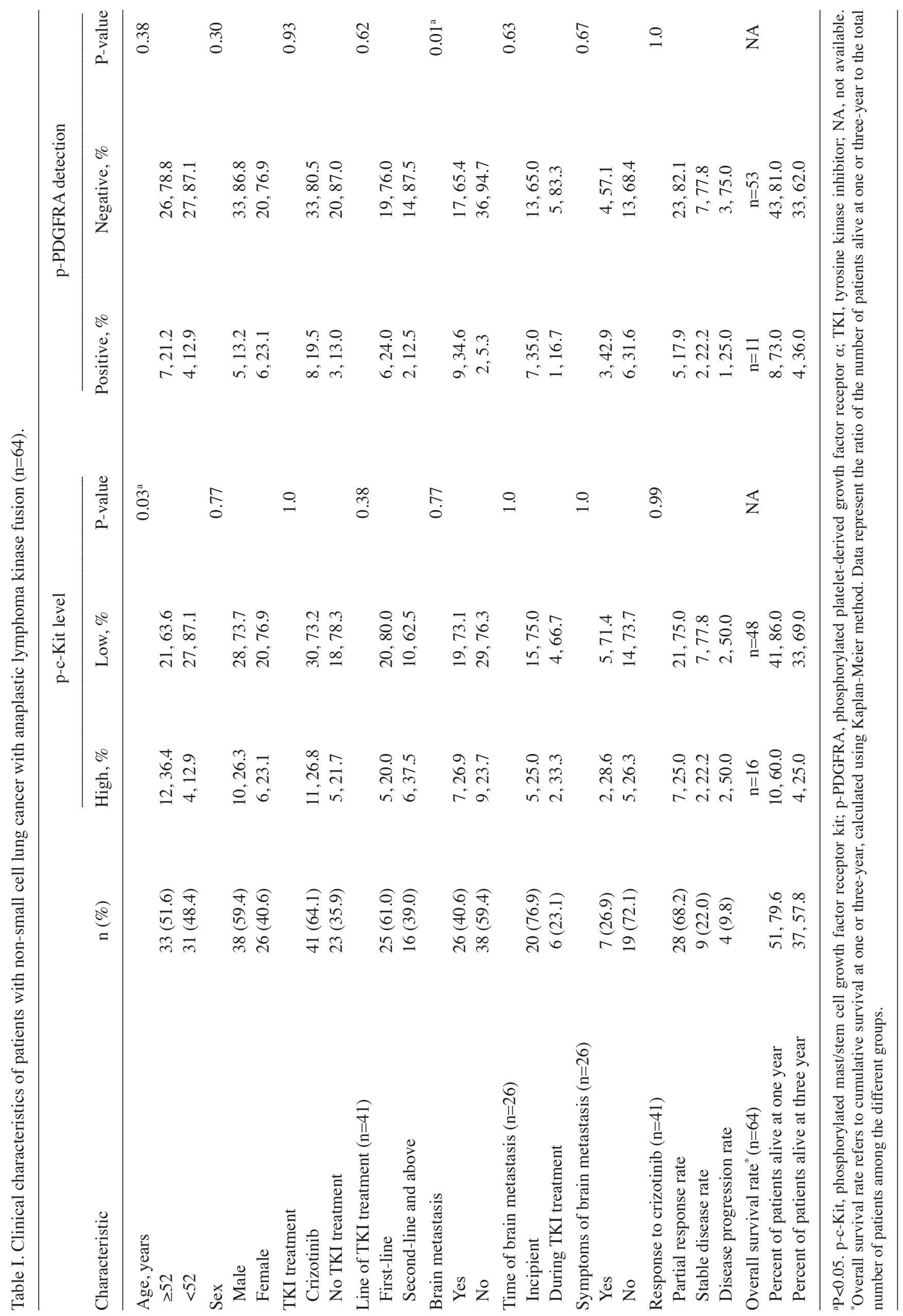


A

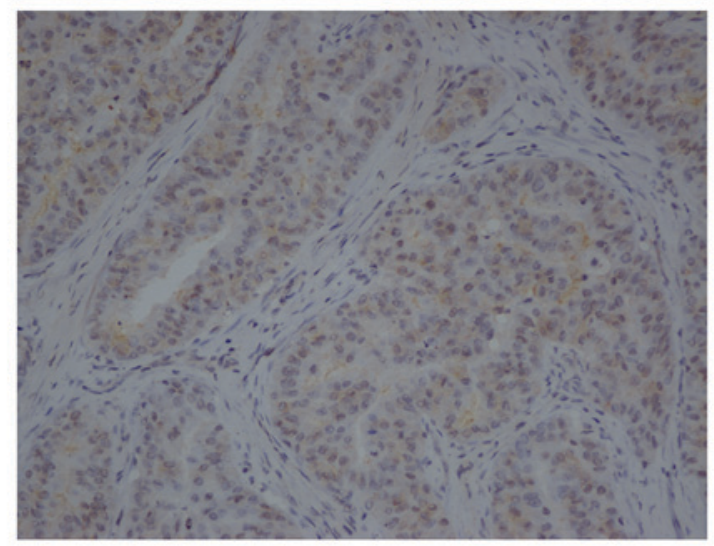

B

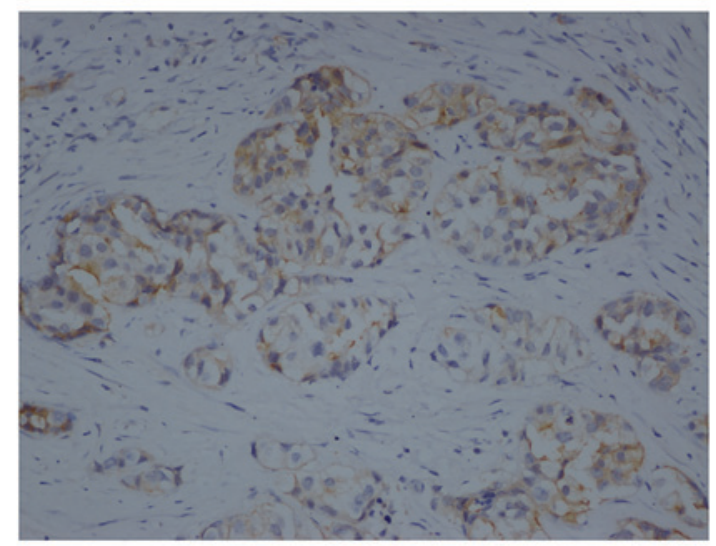

Figure 1. Immunochemical staining in tumor cells. Images of staining of (A) p-c-Kit and (B) p-PDGFRA (magnification, x200). The p-c-Kit protein was mainly expressed in the membrane and cytoplasm of tumor cells, and p-PDGFRA protein was mainly detected in the membrane of tumor cells. p-c-Kit, phosphorylated mast/stem cell growth factor receptor Kit; p-PDGFRA, phosphorylated platelet-derived growth factor receptor $\alpha$.

low-level p-c-Kit groups, as well as the subgroups between p-PDGFRA positive and negative $(\mathrm{P}>0.05)$.

The median PFS time of crizotinib-treated patients $(n=41)$ with ALK fusion was 7 months, (range, 1-44 months; 95\% CI, 5.1-18.5). The median PFS time of the patients with high p-c-Kit levels was significantly shorter than those with low levels (5 vs. 26 months; $\mathrm{P}=0.005$; Fig. 2A). Furthermore, the patients with high levels of p-c-Kit in their samples exhibited significantly lower OS times than those with low-level p-c-Kit (median OS time, 17 months vs. NA, not available; $\mathrm{P}=0.002$; Fig. 2B), whether they had received TKI or not. The one- and three-year OS rates in the patients with high p-c-Kit levels were 60 and $25 \%$, respectively, and in those with low levels they were 86 and $69 \%$ respectively (Table I). Notably, the results revealed no significant difference in PFS (Fig. 3A) of crizotinib and OS (Fig. 3B) between patients with p-PDGFRA-positive tumors and those with p-PDGFRA-negative tumors $(\mathrm{P}>0.05)$.

A multivariate Cox proportional hazard model was used to analyze the significance of p-c-Kit expression in the PFS time of patients. The factors included in the analysis were age, brain metastasis, crizotinib line of treatment and p-c-Kit status of the tumor. The results revealed that a high p-c-Kit level was the only significant predictive factor for poor PFS time in the patients treated by crizotinib $(\mathrm{HR}=2.7$; 95\% CI, 1.0-7.4; $\mathrm{P}=0.048)$. This was also the only significant prognostic factor for poor OS time $(\mathrm{HR}=5.3$; 95\% CI, 1.5-18.5; $\mathrm{P}=0.01$; data not shown).

\section{Discussion}

To date, a limited number of biomarkers have been proven to be associated with survival in patients with ALK fusion NSCLC $(8,9)$. c-Kit is a transmembrane tyrosine kinase, with SCF as a ligand, serving an important role in inducing a number of signal transduction pathways, including those of mitogen-activated protein kinase and phosphatidyl inositol 3-kinase/protein kinase B $(10,11)$. To the best of our knowledge, the present study is the first to investigate the association between the phosphorylated functional proteins of the c-Kit/PDGFRA signaling pathway, and the efficacy of crizotinib and prognosis in patients with advanced ALK fusion NSCLC. The present data revealed that high levels of p-c-Kit occurred more frequently in older patients ( $\geq 52$ years) and p-c-PDGFRA expression was associated with metastasis to the brain $(\mathrm{P}<0.05)$. In the present study, $\mathrm{p}$-PDGFRA expression was detected in primary tumor samples prior to initial treatment in patients with brain metastasis. PDGF serves an important role as a driver of tumor growth in glioblastoma multiforme and other malignant brain tumors, including malignant peripheral nerve sheath tumors and seminoma, and chromosomal region 4q12, containing the PDGFRA and KIT genes, is often amplified in these tumors (12). It is therefore hypothesized that the PDGFRA pathway is associated with a subset of brain metastases occurring in patients with ALK fusion NSCLC, as in the case of the aforementioned patient with PDGFRA amplification in the metastatic brain tumor. However, the intratumoral heterogeneity may be caused by one clone harboring two gene aberrations or two distinctive clones occurring in these patients (13).

Currently, first or second generation TKIs are prescribed to patients with ALK fusion NSCLC as a first-line treatment. The second-generation TKIs could achieve improved PFS (but not OS) times and control of metastatic brain tumors as first-line treatment, compared with crizotinib (14). However, it is unknown which patients would benefit more from the second-generation TKIs. To date, only BIM deletion polymorphism and $E M L 4-A L K$ variants $3 \mathrm{a} / \mathrm{b}$ have been found to be associated with poor clinical response of patients with ALK fusion NSCLC to crizotinib $(8,9)$. Therefore, further studies of the intrinsic mechanism of resistance to crizotinib would be beneficial for improved decision-making in TKI selection for first-line treatment.

In the present study, although no association was observed between the levels of p-c-Kit or p-PDGFRA and the response rate of the tumor following crizotinib treatment, the patients with high p-c-Kit levels exhibited a lower PFS time than those with low levels (5 vs. 26 months, $\mathrm{P}=0.005$ ). Additionally, high levels of p-c-Kit was the only independent prognostic factor for poor PFS time following crizotinib treatment $(\mathrm{HR}=2.7, \mathrm{P}<0.05)$. Although the study has some limitations due to small sample numbers and uncertainty of interpretation of the immunochemical staining results, 
A

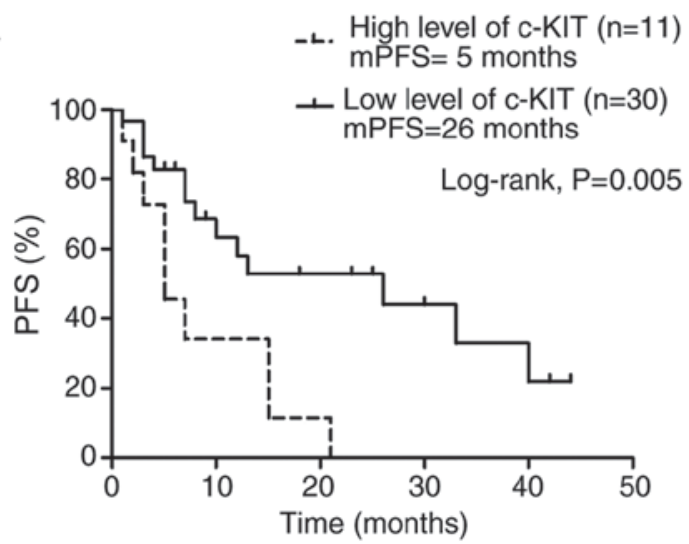

B

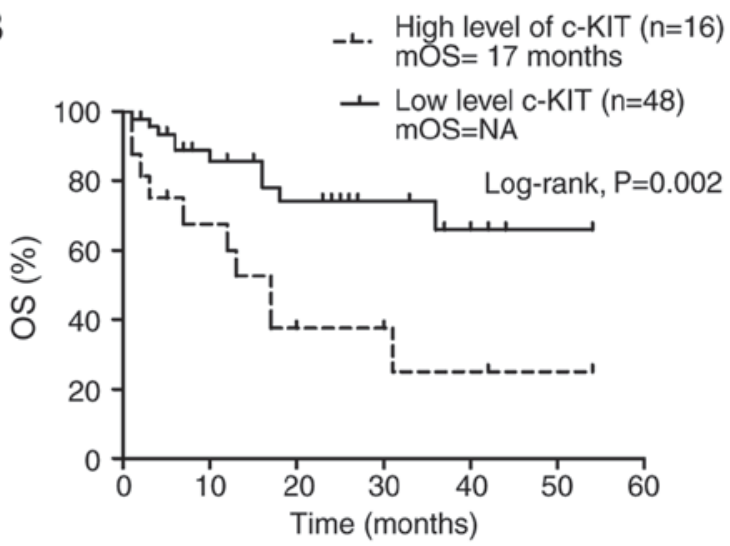

Figure 2. The PFS and OS rates of patients with non-small cell lung cancer with anaplastic lymphoma kinase fusion. Kaplan-Meier survival analyses of patients with high vs. low p-c-Kit levels in their tumor samples. (A) Patients with high levels of p-c-Kit had significantly poorer PFS time (log-rank test, $\mathrm{P}=0.005)$. (B) Patients with high levels of p-c-Kit had significantly poorer OS time (log-rank test, $\mathrm{P}=0.002)$. PFS, progression-free survival; OS, overall survival; p-c-Kit, phosphorylated mast/stem cell growth factor receptor Kit.
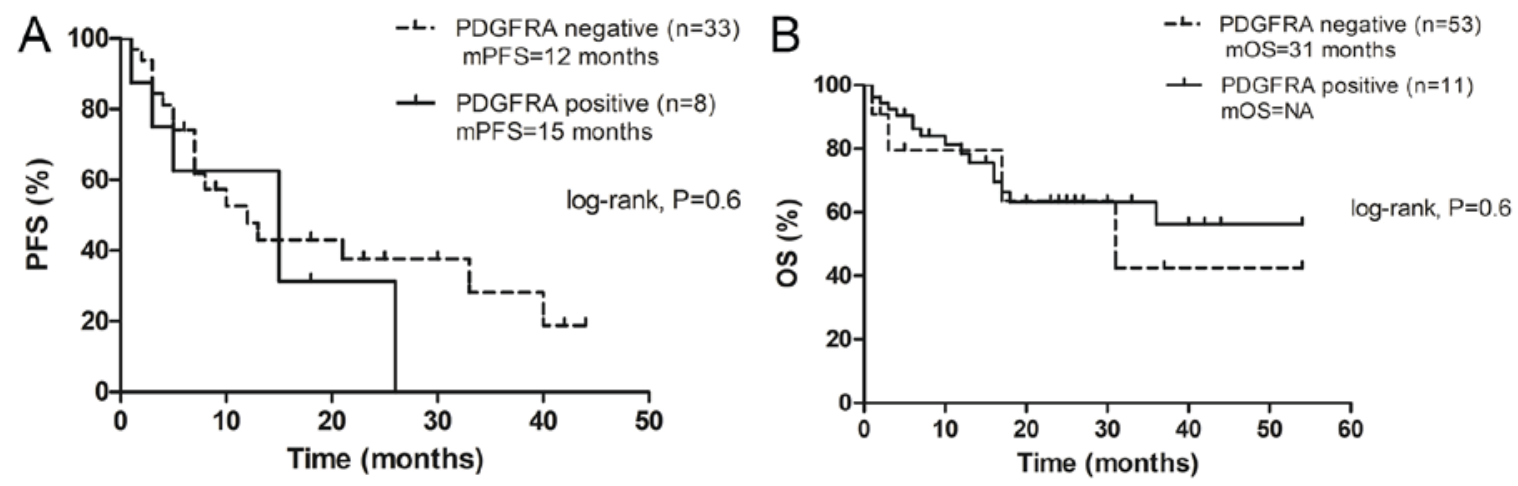

Figure 3. The PFS and OS rates of patients with non-small cell lung cancer with anaplastic lymphoma kinase fusion. Kaplan-Meier survival analysis of patients with p-PDGFRA-positive vs. -negative tumor samples. (A) No significant difference was observed between the PFS time of patients with detectable p-PDGFRA expression compared with those without (log-rank test, $\mathrm{P}=0.6$ ). (B) No significant difference was observed between the OS time of patients with detectable p-PDGFRA expression compared with those without (log-rank test, $\mathrm{P}=0.6$ ). PFS, progression-free survival; OS, overall survival; p-PDGFRA, phosphorylated platelet-derived growth factor receptor $\alpha$.

patients with low levels of p-c-Kit responded significantly better to crizotinib treatment compared with the patients treated by crizotinib reported in the previous clinical trials in which the median PFS was 11 months $(15,16)$. Previous studies identified that the production of high levels of c-Kit in NSCLC tumors is mediated by KIT gene amplification, and not a gene mutation, which stimulates the activation of downstream signaling molecules in the KIT signaling pathway (17). This form of intrinsic crizotinib resistance could be overcome with a combination of ALK and c-Kit inhibitors. However, KIT gene amplification has been reported to occur in $\leq 5 \%$ of NSCLC cases (4). Therefore, it was speculated that if high $\mathrm{p}-\mathrm{c}-\mathrm{Kit}$ expression is caused by overamplification of the KIT gene in only a small subset of ALK fusion tumors, post-transcriptional or post-translational regulation may also contribute to the final majority of p-c-Kit levels. For ALK fusion NSCLC with de novo bypass pathway activation, it is unknown if the next-generation ALK TKIs will be superior to crizotinib.

As the present findings revealed that $\mathrm{p}-\mathrm{c}-\mathrm{Kit}$ levels are a predictive marker for TKI treatment, it was hypothesized that they may also be a prognostic factor for survival in patients with ALK fusion NSCLC. The results identified that high levels of p-c-Kit predicted poor survival of the patients whether they had received TKI treatment or not.

KIT gene functional mutations may be driver mutations in certain malignant tumors, including gastrointestinal stromal tumors, leukemia, lymphoma and mast cell tumors (17). In lung cancer, $69.2 \%$ of small cell lung cancer (SCLC) was reported to express c-Kit, as detected by IHC (18). Conflicting results have been reported on the association between c-Kit expression and prognosis in SCLC $(19,20)$. Studies on c-Kit in NSCLC have focused more on the early stages of the cancer, and the expression of c-Kit in NSCLC tumors has been associated with an increased mortality rate (21). Inconsistent results from previous studies regarding the association between c-Kit expression and prognosis may be due to differences in samples and IHC staining measurements $(10,21)$. In the present study, c-Kit protein was expressed in $46.9 \%$ of advanced NSCLC with ALK fusion, in agreement with a previous study on early stage NSCLC (8). The patients were further divided into two groups (high vs. low levels of c-Kit). The results demonstrated that patients with high levels of p-c-Kit exhibited significantly lower survival times than those with low levels $(\mathrm{P}<0.05)$. Furthermore, high levels 
of p-c-Kit was the only significant indicator of poor survival in patients with ALK fusion NSCLC $(\mathrm{HR}=5.3, \mathrm{P}<0.05)$.

In conclusion, the present study suggests that p-PDGFRA may be expressed more often in ALK-positive cases of NSCLC with brain metastasis, and that c-Kit signaling activation may serve as a predictor of crizotinib efficacy and as a prognostic indicator for advanced stage ALK fusion NSCLC.

\section{Acknowledgements}

Not applicable.

\section{Funding}

No funding was received.

\section{Availability of data and materials}

All data generated or analyzed during the present study are included in this published article.

\section{Authors' contributions}

HY, FW and DH were responsible for collecting and analyzed the data. QD and DX were responsible for immunohistochemical staining. PH and XL analyzed the stained specimens. All authors including HY, FW, DH, QD, DX, PH and XL have read and approved the final version of this manuscript.

\section{Ethics approval and consent to participate}

The present study was approved by the Institutional Review Board of The First Affiliated Hospital, Guangzhou Medical University. Written informed consent was obtained from all participants prior to the study.

\section{Patient consent for publication}

Not applicable.

\section{Competing interests}

The authors declare that they have no competing interests.

\section{References}

1. Zhou JX, Yang H, Deng Q, Gu X, He P, Lin Y, Zhao M, Jiang J, Chen $\mathrm{H}$, Lin Y, et al: Oncogenic driver mutations in patients with non-small-cell lung cancer at various clinical stages. Ann Oncol 24: 1319-1325, 2013.

2. Auliac JB, Monnet I, Dubos-Arvis C, Chiappa AM, Baize N, Bota S, Vergnenegre A, Doubre H, Locher C, Bizieux A, et al: Non-small-cell lung cancer (NSCLC) harboring ALK translocations: Clinical characteristics and management in a real-life setting: A French retrospective analysis (GFPC 02-14 study). Target Oncol 12: 833-838, 2017.

3. Katayama R, Shaw AT, Khan TM, Mino-Kenudson M, Solomon BJ, Halmos B, Jessop NA, Wain JC, Yeo AT, Benes C, et al: Mechanisms of acquired crizotinib resistance in ALK-rearranged lung Cancers. Sci Transl Med 4: 120ra17, 2012.

4. Ramos AH, Dutt A, Mermel C, Perner S, Cho J, Lafargue CJ, Johnson LA, Stiedl AC, Tanaka KE, Bass AJ, et al: Amplification of chromosomal segment 4q12 in non-small cell lung cancer. Cancer Biol Ther 8: 2042-2050, 2009.
5. Kassis ES, Vaporciyan AA, Swisher SG, Correa AM, Bekele BN, Erasmus JJ, Hofstetter WL, Komaki R, Mehran RJ, Moran CA, et al: Application of the revised lung cancer staging system (IASLC Staging Project) to a cancer center population. J Thorac Cardiovasc Surg 38: 412-418.e1-e2, 2009.

6. Eisenhauer EA, Therasse P, Bogaerts J, Schwarts LH, Sargent D, Ford R, Dancey J, Arbuck S, Gwyther S, Mooney M, et al: New response evaluation criteria in solid tumours: Revised RECIST guideline (version 1.1). Eur J Cancer 45: 228-247, 2009

7. Yang H, Wang W, Zhang Y, Zhao J, Lin E, Gao J and He J: The role of NF-E2-related factor 2 in predicting chemoresistance and prognosis in advanced non-small cell lung cancer. Clin Lung Cancer 12: 166-171, 2011.

8. Zhang L, Jiang T, Li X, Wang Y, Zhao C, Zhao S, Xi L, Zhang S, Liu X, Jia Y, et al: Clinical features of Bim deletion polymorphism and its relation with crizotinib primary resistance in Chinese patients with ALK/ROS1 fusion-positive non-small cell lung cancer. Cancer 123: 2927-2935, 2017.

9. Woo CG, Seo S, Kim SW, Jang SJ, Park KS, Song JY, Lee B, Richards MW, Bayliss R, Lee DH and Choi J: Differential protein stability and clinical responses of EML4-ALK fusion variants to various ALK inhibitors in advanced ALK-rearranged non-small cell lung cancer. Ann Oncol 28: 791-797, 2017.

10. Herpel E, Jensen K, Muley T, Warth A, Schnabel PA, Meister M, Herth FJ, Dienemann H, Thomas M and Gottschling S: The cancer stem cell antigens CD133, BCRP1/ABCG2 and CD117/ c-KIT are not associated with prognosis in resected early-stage non-small cell lung cancer. Anticancer Res 31: 4491-4500, 2011.

11. Marech I, Gadaleta CD and Ranieri G: Possible prognostic and therapeutic significance of c-Kit expression, mast cell count and microvessel density in renal cell carcinoma. Int J Mol Sci 15: 13060-13076, 2014.

12. Holtkamp N, Ziegenhagen N, Malzer E, Hartmann C, Giese A and von Deimling A: Characterization of the amplicon on chromosomal segment 4q12 in glioblastoma multiforme. Neuro Oncol 9: 291-297, 2007.

13. Cai W, Lin D, Wu C, Li X, Zhao C, Zheng L, Chuai S, Fei K, Zhou C and Hirsch FR: Intratumoral heterogeneity of ALK-rearranged and ALK/EGFR coaltered lung adenocarcinoma. J Clin Oncol 33: 3701-3709, 2015.

14. Qin A and Gadgeel S: The current landscape of anaplastic lymphoma kinase (ALK) in non-small cell lung cancer: Emerging 3 treatment paradigms and future directions. Target Oncol 12: 709-718, 2017.

15. Solomon BJ, Mok T, Kim DW, Wu YL, Nakagawa K, Mekhail T, Felip E, Cappuzzo F, Paolini J, Usari T, et al: First-line crizotinib versus chemotherapy in ALK-positive lung cancer. N Engl J Med 371: 2167-2177, 2014.

16. Solomon BJ, Cappuzzo F, Felip E, Blackhall FH, Costa DB, Kim DW, Nakagawa K, Wu YL, Mekhail T, Paolini J, et al: Intracranial efficacy of crizotinib versus chemotherapy in patients with advanced ALK-positive non-small-cell lung cancer: Results from PROFILE 1014. J Clin Oncol 34: 2858-2865, 2016.

17. Liang J, Wu YL, Chen BJ, Zhang W, Tanaka Y and Sugiyama H: The C-kit receptor-mediated signal transduction and tumor-related diseases. Int J Biol Sci 9: 435-443, 2013.

18. Li AS, Sun LN and Zhan ZL: Expression and mutational analysis of c-kit gene in small cell lung cancer. Chin J Cancer Prev Treat 14: 917-919, 2007.

19. Yokouchi H, Nishihara H, Harada T, Ishida T, Yamazaki S, Kikuchi H, Oizumi S, Uramoto H, Tanaka F, Harada M, et al: Immunohistochemical profiling of receptor tyrosine kinases, MED12, and TGF- $\beta$ RII of surgically resected small cell lung cancer, and the potential of c-kit as a prognostic marker. Oncotarget 8: 39711-39726, 2017.

20. Terada T: An immunohistochemical and molecular genetic analysis of KIT and PDGFRA in small cell lung carcinoma in Japanese. Int J Clin Exp Pathol 5: 331-338, 2012.

21. Xiao H, Wang J, Liu Y and Li L: Relative influence of c-Kit expression and epidermal growth factor receptor gene amplification on survival in patients with non-small cell lung cancer. Oncol Lett 8: 582-588, 2014.

This work is licensed under a Creative Commons Attribution-NonCommercial-NoDerivatives 4.0 International (CC BY-NC-ND 4.0) License. 